\title{
AN EQUIVALENCE CRITERION FOR INFINITE PRODUCTS OF CAUCHY MEASURES
}

\author{
KAZUKI OKAMURA
}

\begin{abstract}
We give an equivalence-singularity criterion for infinite products of Cauchy measures under simultaneous shifts of the location and scale parameters. Our result is an extension of Lie and Sullivan's result giving an equivalence-singularity criterion under dilations of scale parameters. Our proof utilizes McCullagh's parameterization of the Cauchy distributions and maximal invariant, and a closed-form formula of the Kullback-Leibler divergence between two Cauchy measures given by Chyzak and Nielsen.
\end{abstract}

\section{INTRODUCTION}

The purpose of this note is to establish an equivalence criterion for two infinite products of Cauchy measures. Let $\left(Z_{n}\right)_{n}$ be independent Cauchy random variables with location $z_{n 1} \in \mathbb{R}$ and scale $z_{n 2}>0$, and $\left(W_{n}\right)_{n}$ be independent Cauchy random variables with location $w_{n 1} \in \mathbb{R}$ and scale $w_{n 2}>0$. We will give a necessary and sufficient condition for the equivalence of the laws of $\left(Z_{n}\right)_{n}$ and $\left(W_{n}\right)_{n}$ in terms of $\left(z_{n 1}, z_{n 2}, w_{n 1}, w_{n 2}\right)_{n}$.

The Cameron-Martin theorem characterizes the directions in which an infinite-dimensional Gaussian measure can be translated under the constraint that the translations preserve equivalence of the original and translated measures. Hajek [6, 5] and Feldman [4] establish a dichotomy between two Gaussian measures, which are equivalent or singular to each other. Bogachev [1, Theorems 2.7.2 and 2.12.9] and Shiryaev [10, Theorem 7.6.5] also deal with this issue. It would be interesting to consider this kind of equivalence-singularity dichotomy for infinite-dimensional measures other than the infinite-dimensional Gaussian measures. Recently, Lie and Sullivan [8] gave a characterization of equivalence of measures for infinite-dimensional Cauchy measures which could be regarded as an analogue of the CameronMartin theorem. However, [8] deals with dilations of the scale parameters only, more specifically, [8] considers the case that all locations are identical with each other, that is, $z_{n 1}=w_{n 1}$ for every $n \geq 1$.

We extend [8] to a more general case. We deal with simultaneous changes of the location and scale parameters. In a manner similar to [8], our proof depends on Kakutani's theorem [7] for the equivalence-singularity dichotomy of infinite product measures. However, our proof is different from [8]. Our proof is simpler and shorter. Indeed, we do not use a Taylor expansion of an

2010 Mathematics Subject Classification. 60G30, 60E07.

Key words and phrases. Cauchy distribution, equivalence of measures, Kakutani dichotomy. 
integral for the scale parameter. Our main tool is McCullagh's parametrization and maximal invariant [9]. We will also use an explicit formula of Kullback-Leibler divergence between two Cauchy distributions obtained by Chyzak and Nielsen [2].

1.1. Framework and main result. Throughout this note, for $z \in \mathbb{C}$, $\operatorname{Re}(z)$ and $\operatorname{Im}(z)$ are the real and imaginary parts of $z$. Let $i$ be the imaginary unit. Let the upper-half plane $\mathbb{H}:=\{x+y i \mid x \in \mathbb{R}, y>0\}$. For $z \in \mathbb{H}$, let $P_{z}$ be the Cauchy distribution with parameter $z$ equipped with McCullagh's parametrization [9], that is, the density function of $P_{z}$ is given by $\frac{\operatorname{Im}(z)}{\pi|x-z|^{2}}$. For an infinite sequence $z=\left(z_{n}\right)_{n}$ in $\mathbb{H}$, we let $\bigotimes_{n} P_{z_{n}}$ be the product probability measure of a sequence of probability measures $\left(P_{z_{n}}\right)_{n}$ on the product space $\mathbb{R}^{\mathbb{N}}$ equipped with the cylindrical $\sigma$-algebra. For two measures $\mu$ and $\nu$ on a common probability space such that $\mu$ is absolutely continuous with respect to $\nu$, we denote the Radon-Nikodym derivative of $\mu$ with respect to $\nu$ by $\frac{d \mu}{d \nu}$. We let $E^{P}$ be the expectation with respect to a probability measure $P$.

Theorem 1.1. For every two infinite sequences $z=\left(z_{n}\right)_{n}$ and $w=\left(w_{n}\right)_{n}$ of $\mathbb{H}$, we have that

(i) If $\sum_{n=1}^{\infty} \frac{\left|z_{n}-w_{n}\right|^{2}}{\operatorname{Im}\left(z_{n}\right) \operatorname{Im}\left(w_{n}\right)}<+\infty$, then, $\bigotimes_{n} P_{z_{n}}$ and $\bigotimes_{n} P_{w_{n}}$ are equivalent, that is, $\bigotimes_{n} P_{z_{n}}$ is absolutely continuous with respect to $\bigotimes_{n} P_{w_{n}}$ and vice versa.

(ii) If $\sum_{n=1}^{\infty} \frac{\left|z_{n}-w_{n}\right|^{2}}{\operatorname{Im}\left(z_{n}\right) \operatorname{Im}\left(w_{n}\right)}=+\infty$, then, $\bigotimes_{n} P_{z_{n}}$ and $\bigotimes_{n} P_{w_{n}}$ are singular to each other.

\section{ProOF}

By adopting a version of Kakutani's theorem as in [10, Corollary 7.6.3] for our framework, we have that

Proposition 2.1. For every two infinite sequences $z=\left(z_{n}\right)_{n}$ and $w=\left(w_{n}\right)_{n}$ of $\mathbb{H}$, we have that

(i) If $\sum_{n=1}^{\infty}-\log E^{P_{z_{n}}}\left[\sqrt{\frac{d P_{w_{n}}}{d P_{z_{n}}}}\right]<+\infty$, then, $\bigotimes_{n} P_{z_{n}}$ and $\bigotimes_{n} P_{w_{n}}$ are equivalent, that is, $\bigotimes_{n} P_{z_{n}}$ is absolutely continuous with respect to $\bigotimes_{n} P_{w_{n}}$ and vice versa.

(ii) If $\sum_{n=1}^{\infty}-\log E^{P_{z_{n}}}\left[\sqrt{\frac{d P_{w_{n}}}{d P_{z_{n}}}}\right]=+\infty$, then, $\bigotimes_{n} P_{z_{n}}$ and $\bigotimes_{n} P_{w_{n}}$ are singular to each other.

Let

$$
\chi(z, w):=\frac{|z-w|^{2}}{\operatorname{Im}(z) \operatorname{Im}(w)} .
$$


Let the Kullback-Leibler divergence be

$$
K\left(P_{z} \mid P_{w}\right):=E^{P_{z}}\left[\log \frac{d P_{z}}{d P_{w}}\right], \quad z, w \in \mathbb{H} .
$$

We remark that this is symmetric, specifically,

$$
K\left(P_{z} \mid P_{w}\right)=K\left(P_{w} \mid P_{z}\right), z, w \in \mathbb{H} .
$$

Proposition 2.2 (Chyzak-Nielsen [2]). For $z, w \in \mathbb{H}$,

$$
K\left(P_{z} \mid P_{w}\right)=\log \left(1+\frac{\chi(z, w)}{4}\right) .
$$

Now we show Theorem 1.1 (i). By Jensen's inequality,

$$
\begin{aligned}
\sum_{n=1}^{\infty}-\log E^{P_{z_{n}}}\left[\sqrt{\frac{d P_{w_{n}}}{d P_{z_{n}}}}\right] & \leq \frac{1}{2} \sum_{n=1}^{\infty} E^{P_{z_{n}}}\left[-\log \frac{d P_{w_{n}}}{d P_{z_{n}}}\right]=\frac{1}{2} \sum_{n=1}^{\infty} K\left(P_{z_{n}} \mid P_{w_{n}}\right) \\
& \leq \frac{1}{8} \sum_{n=1}^{\infty} \chi\left(z_{n}, w_{n}\right)<+\infty .
\end{aligned}
$$

The assertion follows from this and Proposition 2.1 (i).

By [9], the function $\chi$ defined in (2.1) is a maximal invariant for the action of the special linear group $S L(2, \mathbb{R})$ to $\mathbb{H} \times \mathbb{H}$ defined by

$$
A \cdot(z, w):=\left(\frac{a z+b}{c z+d}, \frac{a w+b}{c w+d}\right), \quad A=\left(\begin{array}{ll}
a & b \\
c & d
\end{array}\right) \in S L(2, \mathbb{R}), \quad z, w \in \mathbb{H} .
$$

That is,

$$
\chi(A \cdot z, A \cdot w)=\chi(z, w), \quad A \in S L(2, \mathbb{R}), z, w \in \mathbb{H} .
$$

and it holds that for every $z, w, z^{\prime}, w^{\prime} \in \mathbb{H}$ satisfying that $\chi\left(z^{\prime}, w^{\prime}\right)=\chi(z, w)$, there exists $A \in S L(2, \mathbb{R})$ such that $(A \cdot z, A \cdot w)=\left(z^{\prime}, w^{\prime}\right)$. See Eaton [3, Chapter 2] for more details about maximal invariants.

Lemma 2.3. Let

$$
I(z, w):=\frac{\sqrt{\operatorname{Im}(z) \operatorname{Im}(w)}}{\pi} \int_{\mathbb{R}} \frac{1}{|x-z||x-w|} d x .
$$

Then,

$$
I(A \cdot(z, w))=I(z, w), \quad A \in S L(2, \mathbb{R}), \quad z, w \in \mathbb{H} .
$$

Proof. In this proof we let

$$
A \cdot z:=\frac{a z+b}{c z+d}, \quad A=\left(\begin{array}{ll}
a & b \\
c & d
\end{array}\right) \in S L(2, \mathbb{R}), z \in \mathbb{H} .
$$

Since $|x-z|=|(x+\epsilon i)-(z+\epsilon i)|$ and $\chi$ is invariant, we have that for every $\epsilon>0, x \in \mathbb{R}, z \in \mathbb{H}$,

$$
\frac{\sqrt{\operatorname{Im}(z)}}{|x-z|}=\frac{1}{\sqrt{\epsilon \chi(x+\epsilon i, z+\epsilon i)}}=\frac{1}{\sqrt{\epsilon \chi(A \cdot(x+\epsilon i), A \cdot(z+\epsilon i))}}
$$

If we let $A=\left(\begin{array}{ll}a & b \\ c & d\end{array}\right)$, then,

$$
\operatorname{Im}(A \cdot(x+\epsilon i))=\frac{\epsilon}{(c x+d)^{2}+c^{2} \epsilon^{2}} .
$$


Hence,

$\frac{1}{\sqrt{\epsilon \chi(A \cdot(x+\epsilon i), A \cdot(z+\epsilon i))}}=\frac{\sqrt{\operatorname{Im}(A \cdot(z+\epsilon i))}}{|A \cdot(x+\epsilon i)-A \cdot(z+\epsilon i)| \sqrt{(c x+d)^{2}+c^{2} \epsilon^{2}}}$.

Therefore, by letting $\epsilon \rightarrow+0$, we have that for every $x$ such that $c x+d \neq$ 0 ,

$$
\frac{\sqrt{\operatorname{Im}(z)}}{|x-z|}=\frac{\sqrt{\operatorname{Im}(A \cdot z)}}{|A \cdot x-A \cdot z||c x+d|} .
$$

Therefore by the change-of-variable formula,

$$
I(z, w)=\frac{1}{\pi} \int_{\mathbb{R}} \frac{\sqrt{\operatorname{Im}(A \cdot z) \operatorname{Im}(A \cdot w)}}{|A \cdot x-A \cdot z||A \cdot x-A \cdot w|(c x+d)^{2}} d x=I(A \cdot z, A \cdot w) .
$$

By Lemma 2.3 and [3, Theorem 2.3], we see that there exists a unique function $J:[0, \infty) \rightarrow[0, \infty)$ such that

$$
J(\chi(z, w))=I(z, w), z, w \in \mathbb{H} .
$$

Lemma 2.4. $\lim _{t \rightarrow+\infty} J(t)=0$.

Proof. By [9], we have that for every $t \geq 0$, there exists $\lambda \geq 1$ such that $\chi(\lambda i, i)=t$. As a function of $\lambda, \chi(\lambda i, i)=\frac{(\lambda-1)^{2}}{\lambda}$ is strictly increasing if $\lambda \geq 1$. It holds that for $\lambda \geq 1$,

$$
\frac{\sqrt{\lambda}}{\sqrt{x^{2}+\lambda^{2}}} \frac{1}{\sqrt{x^{2}+1}} \leq \min \left\{1,|x|^{-1 / 2}\right\} \frac{1}{\sqrt{x^{2}+1}}
$$

and $\min \left\{1,|x|^{-1 / 2}\right\} \frac{1}{\sqrt{x^{2}+1}}$ is integrable on $\mathbb{R}$.

Since $\lim _{\lambda \rightarrow \infty} \sqrt{\frac{\lambda}{x^{2}+\lambda^{2}}}=0$, we have that by the dominated convergence theorem,

$$
\lim _{\lambda \rightarrow+\infty} I(\lambda i, i)=\lim _{\lambda \rightarrow+\infty} \frac{1}{\pi} \int_{\mathbb{R}} \frac{\sqrt{\lambda}}{\sqrt{x^{2}+\lambda^{2}}} \frac{1}{\sqrt{x^{2}+1}} d x=0 .
$$

Lemma 2.5. If $\bigotimes_{n} P_{z_{n}}$ and $\bigotimes_{n} P_{w_{n}}$ are equivalent, then, $\left\{\chi\left(z_{n}, w_{n}\right)\right\}_{n}$ is bounded in $[0, \infty)$.

Proof. If $\bigotimes_{n} P_{z_{n}}$ and $\bigotimes_{n} P_{w_{n}}$ are equivalent, then, by Proposition 2.1, we have that

and hence,

$$
\sum_{n=1}^{\infty}-\log E^{P_{z_{n}}}\left[\sqrt{\frac{d P_{w_{n}}}{d P_{z_{n}}}}\right]<+\infty
$$

$$
\lim _{n \rightarrow \infty} J\left(\chi\left(z_{n}, w_{n}\right)\right)=\lim _{n \rightarrow \infty} I\left(z_{n}, w_{n}\right)=\lim _{n \rightarrow \infty} E^{P_{z_{n}}}\left[\sqrt{\frac{d P_{w_{n}}}{d P_{z_{n}}}}\right]=1 .
$$


Now the assertion follows from this and Lemma 2.4.

Lemma 2.6. If $\chi(z, w) \leq C_{1}$, then, there exists a constant $C_{2}$ depending only on $C_{1}$ such that

$$
\frac{1}{C_{2}} \leq \inf _{x \in \mathbb{R}} \frac{\operatorname{Im}(w)}{\operatorname{Im}(z)} \frac{|x-z|^{2}}{|x-w|^{2}} \leq \sup _{x \in \mathbb{R}} \frac{\operatorname{Im}(w)}{\operatorname{Im}(z)} \frac{|x-z|^{2}}{|x-w|^{2}} \leq C_{2} .
$$

Proof. By the definition of $\chi$, we have that there exist $C_{3} \in(1, \infty)$ depending only on $C_{1}$ such that for every $(z, w)$ satisfying $\chi(z, w) \leq C_{1}$,

$$
\frac{1}{C_{3}} \leq \frac{\operatorname{Im}(z)}{\operatorname{Im}(w)} \leq C_{3} \text {. }
$$

We have that

$$
\begin{gathered}
\sup _{x \in \mathbb{R}} \frac{\operatorname{Im}(w)}{\operatorname{Im}(z)} \frac{|x-z|^{2}}{|x-w|^{2}} \leq \frac{\operatorname{Im}(z)}{\operatorname{Im}(w)}+\frac{\operatorname{Im}(w)}{\operatorname{Im}(z)} \sup _{x \in \mathbb{R}} \frac{(x-\operatorname{Re}(z-w))^{2}}{x^{2}+\operatorname{Im}(w)^{2}} \\
\leq \frac{\operatorname{Im}(z)}{\operatorname{Im}(w)}+\frac{\operatorname{Im}(w)}{\operatorname{Im}(z)}\left(1+\frac{\operatorname{Re}(z-w)^{2}}{\operatorname{Im}(w)^{2}}\right) \leq 2 C_{3}+C_{1} .
\end{gathered}
$$

The lower bound follows from the upper bound and symmetry about $z$ and $w$.

Proof of Theorem 1.1 (ii). By Lemma 2.5 and Proposition 2.1, we have that if $\left\{\chi\left(z_{n}, w_{n}\right)\right\}_{n}$ is not bounded, then, $\bigotimes_{n} P_{z_{n}}$ and $\bigotimes_{n} P_{w_{n}}$ are singular to each other. We assume that $\left\{\chi\left(z_{n}, w_{n}\right)\right\}_{n}^{n}$ is bounded.

Let $X_{i}(x):=x_{i}, i \in \mathbb{N}$, for $x=\left(x_{i}\right)_{i} \in \mathbb{R}^{\mathbb{N}}$. Then, $\left\{X_{i}\right\}_{i}$ are independent under $\bigotimes_{n} P_{w_{n}}$. By the assumption of Theorem 1.1 (ii) and (2.2),

$\sum_{n=1}^{\infty} E^{\bigotimes_{n} P_{w_{n}}}\left[\log \frac{d P_{w_{n}}}{d P_{z_{n}}}\left(X_{n}\right)\right]=\sum_{n=1}^{\infty} E^{P_{w_{n}}}\left[\log \frac{d P_{w_{n}}}{d P_{z_{n}}}\right]=\frac{1}{2} \sum_{n=1}^{\infty} K\left(P_{w_{n}} \mid P_{z_{n}}\right)=+\infty$.

By Lemma 2.6 and

$$
\frac{d P_{w}}{d P_{z}}(x)=\frac{\operatorname{Im}(w)}{\operatorname{Im}(z)} \frac{|x-z|^{2}}{|x-w|^{2}}, x \in \mathbb{R}
$$

we have that $\left\{\left|\log \frac{d P_{w_{n}}}{d P_{z_{n}}}\left(X_{n}\right)\right|\right\}_{n}$ is uniformly bounded. Therefore, by the Kolmogorov three series theorem and the Kolmogorov 0-1 law, $\sum_{n=1}^{\infty} \log \frac{d P_{w_{n}}}{d P_{z_{n}}}\left(X_{n}\right)$ diverges with probability one under $\bigotimes_{n} P_{w_{n}}$.

By [10, Theorem 7.6.1], it holds that with probability one under $\bigotimes_{n} P_{w_{n}}$, $\lim _{N \rightarrow \infty} \prod_{n=1}^{N} \frac{d P_{w_{n}}}{d P_{z_{n}}}\left(X_{n}\right)$ exists by allowing the limit takes $+\infty$. By this and the Kolmogorov 0-1 law, it holds that $\bigotimes_{n} P_{w_{n}}\left(\sum_{n=1}^{\infty} \log \frac{d P_{w_{n}}}{d P_{z_{n}}}\left(X_{n}\right)=+\infty\right)=1$ 
or $\bigotimes_{n} P_{w_{n}}\left(\sum_{n=1}^{\infty} \log \frac{d P_{w_{n}}}{d P_{z_{n}}}\left(X_{n}\right)=-\infty\right)=1$. We have that
$\lim _{N \rightarrow \infty} \prod_{n=1}^{N} \frac{d P_{w_{n}}}{d P_{z_{n}}}\left(X_{n}\right)=\frac{d\left(\bigotimes_{n} P_{w_{n}}\right)}{d\left(\bigotimes_{n} P_{z_{n}}\right)}, \bigotimes_{n} P_{w_{n}}$-a.s.

and

$$
\bigotimes_{n} P_{w_{n}}\left(\frac{d\left(\bigotimes_{n} P_{w_{n}}\right)}{d\left(\bigotimes_{n} P_{z_{n}}\right)}=0\right)=0
$$

Hence,

$$
\bigotimes_{n} P_{w_{n}}\left(\frac{d\left(\bigotimes_{n} P_{w_{n}}\right)}{d\left(\bigotimes_{n} P_{z_{n}}\right)}=+\infty\right)=\bigotimes_{n} P_{w_{n}}\left(\sum_{n=1}^{\infty} \log \frac{d P_{w_{n}}}{d P_{z_{n}}}\left(X_{n}\right)=+\infty\right)=1 .
$$

By [10, Theorem 7.6.2], we have the assertion.

Remark 2.7. We can also show Theorem 1.1 by using [8]. Let $\lambda_{n}=$ $\lambda_{n}\left(z_{n}, w_{n}\right)$ be the number such that $\chi\left(z_{n}, w_{n}\right)=\chi\left(\lambda_{n} i, i\right)$. Then, by (2.4), $I\left(z_{n}, w_{n}\right)=I\left(\lambda_{n} i, i\right)$. By [8, Theorems 1.1 and 2.1],

$$
\begin{gathered}
\sum_{n=1}^{\infty}-\log I\left(\lambda_{n} i, i\right)<+\infty \Longleftrightarrow \sum_{n=1}^{\infty}\left(\lambda_{n}-1\right)^{2}<+\infty \\
\Longleftrightarrow \sum_{n=1}^{\infty} \frac{\left|z_{n}-w_{n}\right|^{2}}{\operatorname{Im}\left(z_{n}\right) \operatorname{Im}\left(w_{n}\right)}=\sum_{n=1}^{\infty} \frac{\left(\lambda_{n}-1\right)^{2}}{\lambda_{n}}<+\infty .
\end{gathered}
$$

Acknowledgement. The author is supported by JSPS Grant-in-Aid 19 K14549.

\section{REFERENCES}

1. Vladimir I. Bogachev, Gaussian measures, Mathematical Surveys and Monographs Vol.62, American Mathematical Society, 1998.

2. Frederic Chyzak and Frank Nielsen, A closed-form formula for the Kullback-Leibler divergence between Cauchy distributions, preprint, available at arXiv 1905.10965v2.

3. Morris L. Eaton, Group invariance applications in statistics, Regional Conference Series in Probability and Statistics, Institute of Mathematical Statistics, 1989.

4. Jacob Feldman, Equivalence and perpendicularity of Gaussian processes, Pacific Journal of Mathematics 8 (1958), 699-708.

5. Jaroslav Hajek, On a property of normal distributions of any stochastic process, Czechoslovak Mathematical Journal 8 (1958), 610-618.

6. - A property of J-divergence of marginal probability distributions, Czechoslovak Mathematical Journal 8 (1958), 460-463.

7. Shizuo Kakutani, On equivalence of infinite product measures, Annals of Mathematics 49 (1948), 214-224.

8. Han Cheng Lie and Tim J. Sullivan, Quasi-invariance of countable products of Cauchy measures under non-unitary dilations, Electronic Communications in Probability 23 (2018), no. 8, 1-6.

9. Peter McCullagh, On the distribution of the Cauchy maximum-likelihood estimator, Proceedings of the Royal Society. London. Series A 440 (1993), 475-479.

10. Albert N. Shiryaev, Probability-2, 3rd ed., Springer, 2018.

School of General Education, Shinshu University, 3-1-1, Asahi, Matsumoto, NAGANO, 390-8621, JAPAN.

Email address: kazukio@shinshu-u.ac.jp 